\title{
The dynamical design of a steel frame based on the arbitrary Lagrange-Euler method
}

\author{
W. Xu, G. Chen, Z. Deng, X. Huang \& X. Chen \\ Institute of Structural Mechanics, CAEP, PRC
}

\begin{abstract}
A steel frame was one of the key components of the drop tower. With the steel frame's guide, the sample could be dropped from a given height with a position considered, and the steel frame was decelerated with its kinetic energy absorbed with the flow of water in the buffer. The drop tower demanded that the steel frame must be 5.0 meters long, which would reduce its stiffness and the impact load would change with the variety of the steel frame structure. In order to take the change of impact load into consideration, the water flow was taken into the analysis model by the arbitrary Lagrange-Euler method. The design principle was built by the dynamical analysis of steel impact on the buffer. With the numbers of numerical analysis of the designed structure, the final steel frame with high impact load resistance was gained.

Keywords: gliding steel frame, impact, design.
\end{abstract}

\section{Introduction}

A drop tower is the key test equipment to be mainly used to study dynamical mechanical behaviors such as deformation of the productions, in order to assess the productions' environmental suitability when dropping from a high altitude to a target. Currently, there are a lot of drop towers, such as MPM's drop tower systems which can be configured to satisfy ASTM standards. But the drop heights are not higher than ten meters. Only a few super-high drop towers can be found in the world, such as two of CAEP (China Academy of Engineer and Physics) Tower (No. 1: about $20 \mathrm{~m}$ in height and $2.5 \mathrm{~m}$ in span; No. 2: about $60 \mathrm{~m}$ in height and $5 \mathrm{~m}$ in span), SNL Tower (about $54 \mathrm{~m}$ in height and $1 \mathrm{~m}$ in span) and LLNL Tower (Site-300: about 30m in height). There are arch-bridge-like gliding 
steel frame in the pre-three towers and no gliding steel frame in the Site-300. The aim of paper is to design the gliding steel frame of No. 2 of CAEP.

The principle of the drop tower is that: sample was installed with a given gesture on the gliding steel frame, then elevated to test height and released. Sample dropped with the glide of steel frame. Sample separated from steel frame at a given height and impacted on target, and steel frame impacted on buffer. Steel frame is one of the key components of the tower. The usual method of the steel frame design is based on theory. The impact load acting on steel frame was gained by experimental formulae according to frame mass and buffer structure. Obviously, the frame stiffness was ignored from the above method, which was suitable to frame whose span was shorter and height was lower. When steel frame span became longer, the stiffness of frame became more flexible. And the impact load varied with the change of frame structure. In order to take the change of load into considered, the paper integrated the buffer into the numerical simulation model, and designed the frame structure based on the impact analysis.

\section{Structure impact analysis with flow-structure-interaction}

During the structure impact, the difference between material properties such as stiffness will arouse hourglass and make the contact invalid, which will affect the analysis proceeding and the numerical results' precision. In order to increase the analysis precision, the paper used the surface to surface contact with eroding method and segment-based penalty algorithm. The single point integrate method was replaced by volume integrate method, and the hourglass control algorithm was introduced to the numerical model, which were used to reduce the hourglass deformation and hourglass energy.

The frame kinetic energy was absorbed with the flow of water in the buffer. The water element based on Lagrange would distort, which maybe influence the analysis proceeding and precision. So the Euler method was used to simulate the water's large deformation, because the Euler element can keep non-deforming. The solid structures were still Lagrange elements. The interaction between flow and structures was simulated by the arbitrary Lagrange-Euler (ALE) algorithm. Above all, a numerical method was build, which could calculate impact load according to the frame structure property.

A Lagrange time step was performed firstly in ALE time step, followed by an advection step. In advection step, the node to move was decided, and then boundary node was moved followed by interior node's movement. The last step was calculating the transport of element-centered variables and calculating the momentum transport and updating the velocities.

\section{Finite element model}

Because structure and load have two orthogonal symmetry planes, the finite element model (Figure 1) with air mesh was quarter of the total structure in order to reduce the calculating scale, which was meshed by hexahedron element. The 


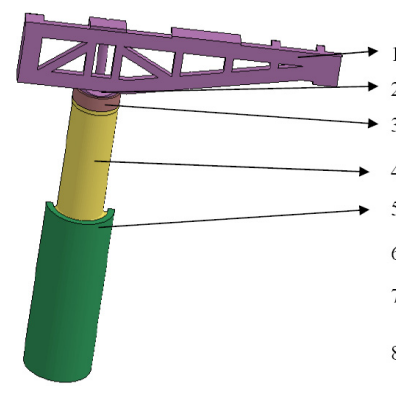

a) View 1

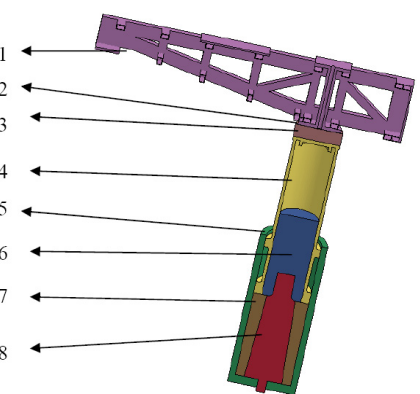

b) View 2

Figure 1: Finite element model: (1-gliding frame; 2-impact; 3-energy absorbing; 4-piston; 5-cylinder block; 6-air; 7-water; 8-load-off cone).

air model was used to model the water's flowing into. So water elements and air elements were multi-material elements based on the Euler algorithm.

\section{Material model of steel}

The dynamical properties of materials were different from the static properties, which would be affected by the material particle inertia. The rate-depended strain-stress curves (Figure 2) were gained by Hopkinson tensile bar (Figure 3),

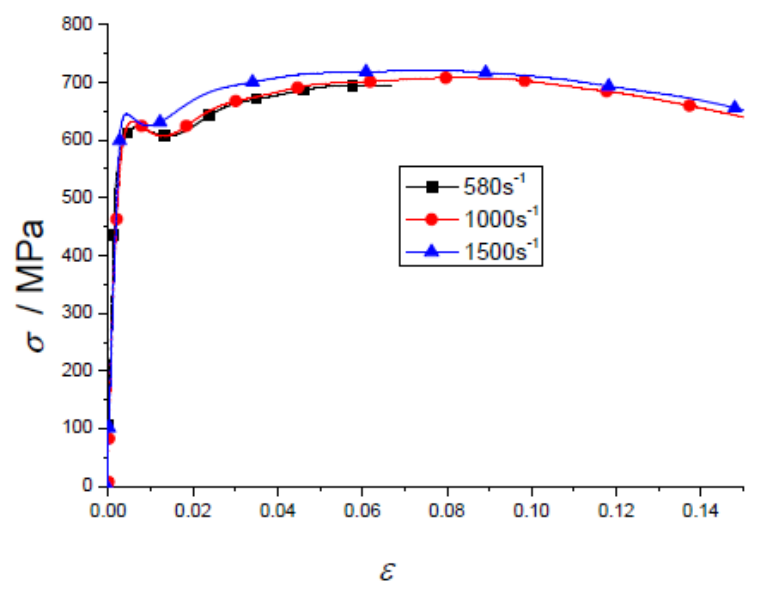

Figure 2: Stress-strain curves via different strain rates. 
from which the plastic-kinematic model parameters [1] (Formula (1), Table 1) were gained to model isotropic and kinematic hardening plasticity including rate effects with static stress-strain curve.

$$
\sigma_{y}=\left(\sigma_{0}+\beta E_{p} \varepsilon_{e f f}^{p}\right)\left[1+\left(\frac{\dot{\varepsilon}}{C}\right)^{\frac{1}{p}}\right]
$$

Table 1: Parameters of constitutive model.

\begin{tabular}{|c|c|c|c|c|}
\hline $\begin{array}{c}\rho \\
\mathrm{g} / \mathrm{mm}^{3}\end{array}$ & $\begin{array}{c}E \\
\mathrm{MPa}\end{array}$ & $\boldsymbol{v}$ & $\begin{array}{c}\sigma_{0} \\
\mathrm{MPa}\end{array}$ & $\begin{array}{c}E_{p} \\
\mathrm{MPa}\end{array}$ \\
\hline 0.0078 & 200000 & 0.28 & 390 & 958 \\
\hline$\beta$ & $C$ & $p$ & $\varepsilon_{f}$ & - \\
\hline 0 & 1 & -0.6 & 0.15 & - \\
\hline
\end{tabular}

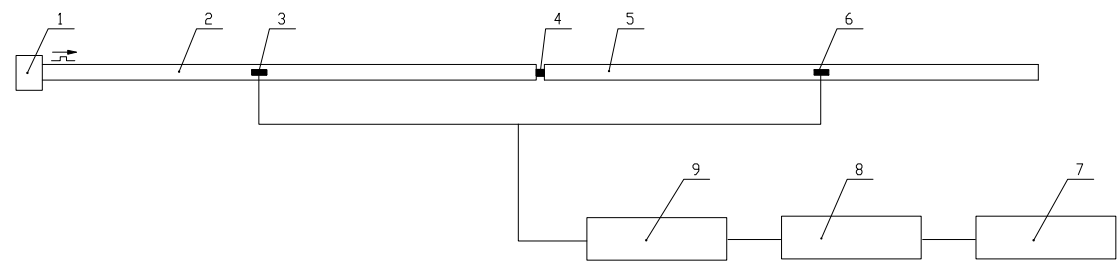

Figure 3: Split Hopkinson tensile bar: (1-incident wave generator; 2-incident bar; 3-strain gauge; 4-sample; 5-transmit bar; 6- strain gauge; 7-computer; 8-oscilloscope; 9-amplifier).

\section{Design of gliding steel frame}

The frame member of gliding steel frame (GSF) was square tubing in order to reduce the mass of GSF and the impact load acting on GSF. When impact load acting on GSF, the middle of GSF would deflect and vibrated. The local stress of interface or joint would be beyond the yield stress, which would affect the safety. This was the key factor of the structure designing.

If defining half of GSF (HGSF) as three concentrated mass, and the mass centers such as F1 F2 and F3 were located as Figure 5. If the center was the fulcrum, the HGSF would not deflect when the moments of F1, F2 and F3 keep balance. Because the sum of L2 and L3 was larger than L1, the mass of M3 and M2 should be lighter than M1. Based on above analysis, GSF should be design as arch bridge. And the M1 should be more weight to adjust the gravity center of HGSF closed to impact center. 


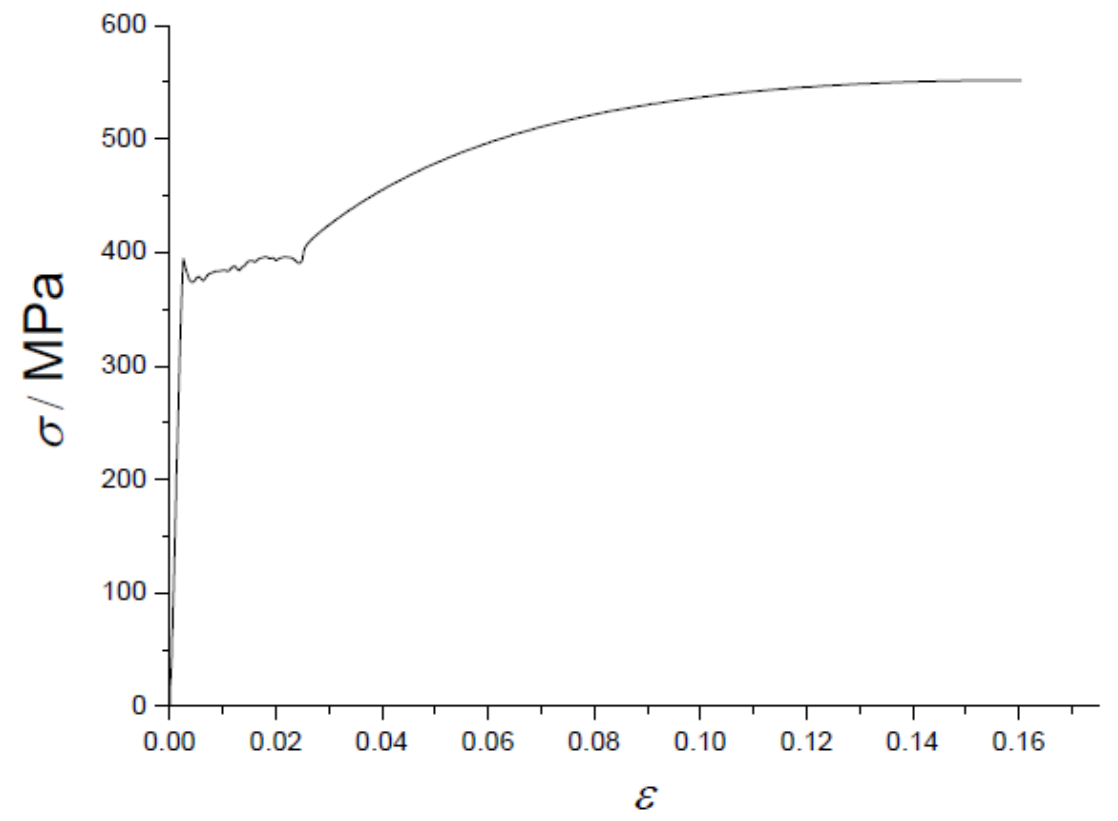

Figure 4: $\quad$ Static stress-strain curve $\left(\dot{\varepsilon}=0.0007 s^{-1}\right)$.

After a few modification and analysis based on impact analysis, the GSF final structure gained as Figure 6. The numerical analysis results were shown in Figure 7 and Figure 8. Figure 7 indicated that: the moment when velocity of point $\mathrm{B}$ was equal to that of point $\mathrm{c}$ was earlier than that of the moment when velocities of point $A$ and point $C$ were equal. The velocity of point $B$ descended fast than that of point $\mathrm{A}$, and bounced at about $5 \mathrm{~ms}$, then bounced to maximum velocity at about $6.9 \mathrm{~ms}$, final was equal to point A velocity at $14.7 \mathrm{~ms}$. But the velocities would not keep equal, but changed further more. The stress emerged when impacted. Initially, the maximum stress was located near impact. With the development of impact and deformation, the maximum stress moved to the middle of GSF from the end of GSF. The impact test illustrated GSF remained elastic.

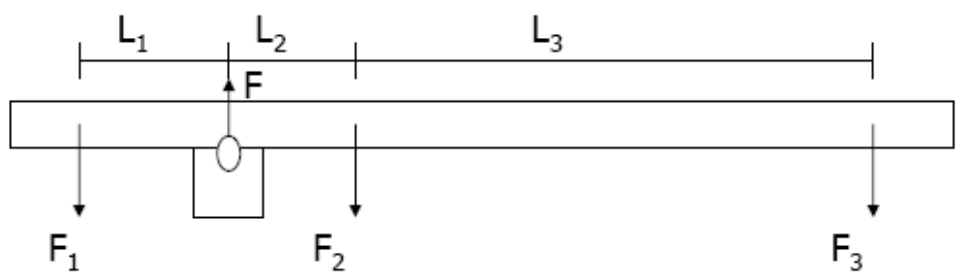

Figure 5: Force state of HGSF (half of gliding steel frame). 


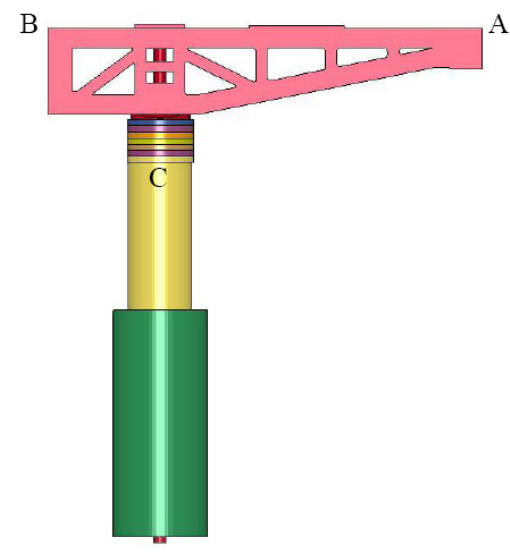

Figure 6: GSF final structure and its analysis model.

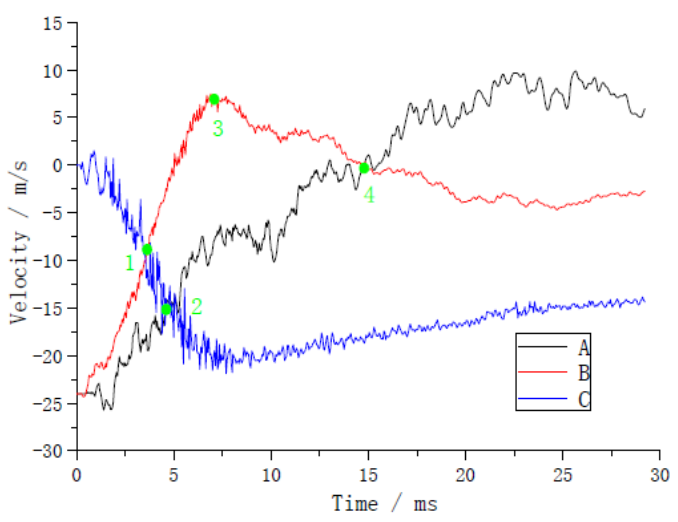

Figure 7: Velocity curves of A, B and C in Figure 6.

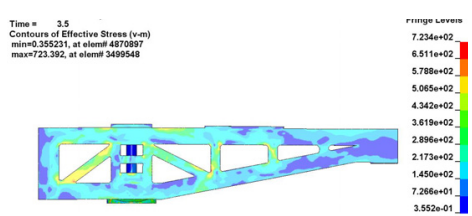

a) $\mathrm{t}=3.50 \mathrm{~ms}$

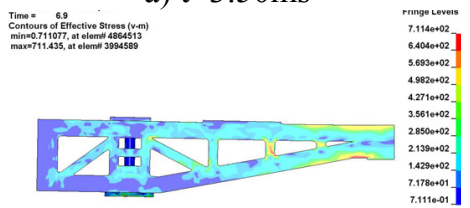

c) $\mathrm{t}=6.90 \mathrm{~ms}$

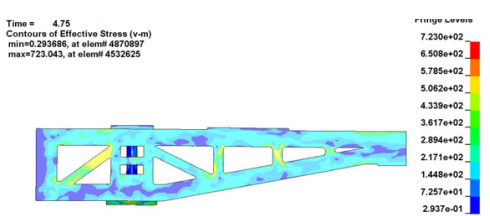

b) $\mathrm{t}=4.75 \mathrm{~ms}$

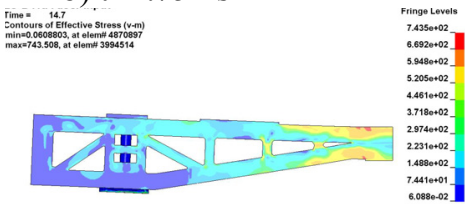

d) $\mathrm{t}=14.70 \mathrm{~ms}$

Figure 8: Stress states of key moments in Figure 7 (MPa). 


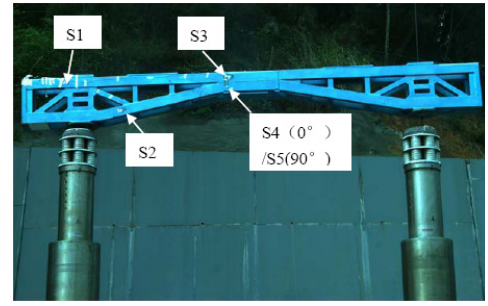

Figure 9: Impact test and strain measuring points.

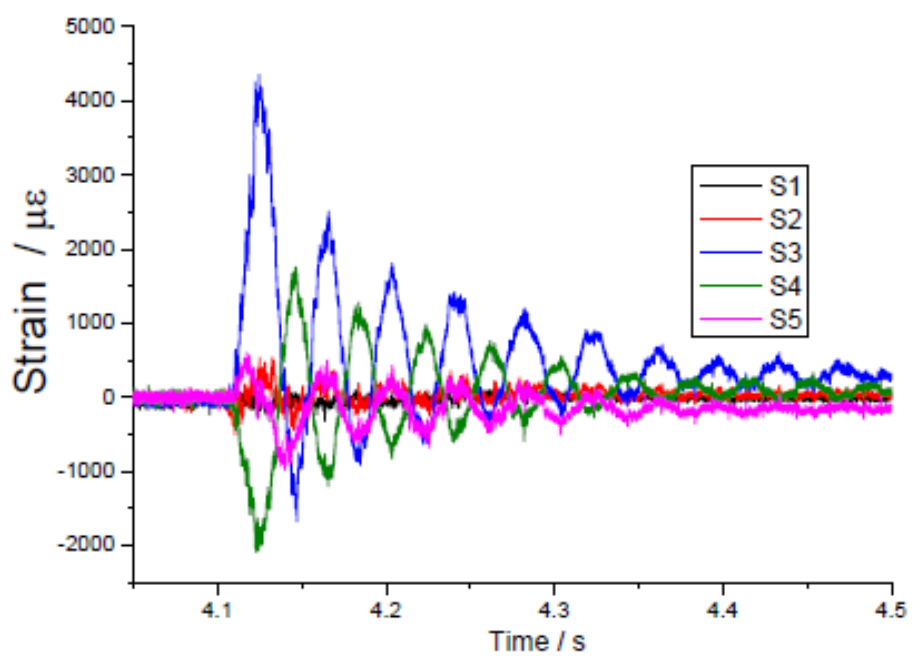

Figure 10: Strain curves of measure points in Figure 9.

\section{Summary}

Based on impact numerical method with flow-structure interaction, the principle that the gravity center of HGSF should be closed to the axial of impact were gained. Connected with the impact test, the GSF structure was created.

\section{Reference}

[1] LS-DYNA keywords user's manual [M], 2007. 